\section{Vorteile für die Tonsillektomie mit bipolarer Schere}

Wie sich das Kühlen des Rachens nach Tonsillektomie mit einer bipolaren Schere auf den postoperativen Schmerz auswirkt, hat eine japanische Studie untersucht. Vergleichsverfahren war die traditionelle "kalte" Dissektion. Die Resultate sprechen stark für die Bipolarschere.

n die Studie an der HNO-Klinik der Universität Osaka waren 189 Patienten einbezogen, von denen 79 mit einer bipolaren Schere operiert wurden. Anschließend kühlten die Chirurgen den Rachen während zehn Minuten mit $500 \mathrm{ml}$ einer $4{ }^{\circ} \mathrm{C}$

kalten Salzlösung, die epipharyngeal wieder abgesaugt wurde.110 Patienten unterzogen sich dem traditionellen Operationsverfahren mit stumpfem Dissektor und Schlinge; dies gilt als die Methode, bei der die geringsten postoperativen Schmerzen auftreten.

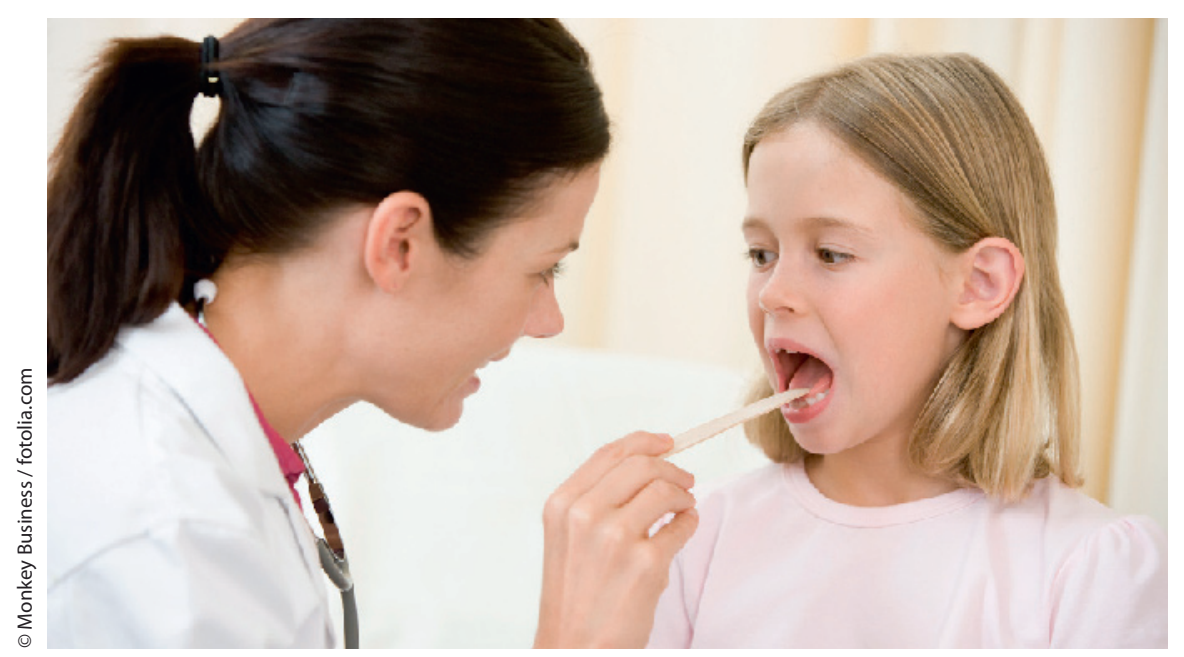

Rezidivierende antibiotikapflichtige Tonsillitiden können eine Tonsillektomie erforderlich machen. Dafür stehen mehrere Verfahren zur Auswahl.

\section{Wie sicher ist die ambulante Thyreoidektomie?}

Die ambulante Thyreoidektomie scheint praktikabel und sicher zu sein. Allerdings hat sich gezeigt, dass es bestimmte Patientengruppen mit erhöhtem postoperativem Betreuungsbedarf gibt: Männer und Patienten mit Morbus Basedow.

U m Sicherheit und Machbarkeit der ambulant vorgenommenen Thyreoidektomie zu überprüfen, wurden retrospektiv die Daten aller Thyreodektomiepatienten eines spezialisierten Versorgungszentrums zwischen Januar 2004 und März 2010 ausgewertet.

Von insgesamt 148 Patienten wurde bei 79 Patienten eine Teilresektion oder eine Hemithyreoidektomie vorgenommen. Diese Gruppe wurde mit 72 Patienten verglichen, bei denen die Schilddrüse er- gänzend operiert oder vollständig entfernt worden war.

Bei 12,5\% der Patienten mit totaler Thyreoidektomie war eine nicht geplante stationäre Betreuung über Nacht notwendig, bei den hemithyreoidektomierten war dies dagegen nur bei 5,1\% der Fall. Grund war meist die Behandlung von Schmerzen oder Übelkeit, und diese Beschwerden traten überwiegend bei Männern (32 vs. $6 \%)$ und bei Patienten mit Morbus Basedow (36 vs. $6 \%$ ) auf.
Im Resultat erwies sich das Kühlverfahren als sehr effektiv. Die Schmerzgrade lagen während der ersten Woche postoperativ in der Gruppe der Patienten, die mit der bipolaren Schere operiert worden waren, signifikant unter jenen der stumpf Operierten. Zur Messung verwendeten die Forscher eine visuelle Analogskala. Der intraoperative Blutverlust fiel naturgemäß ebenfalls zugunsten der Schere aus: Verloren die Patienten hier im Mittel $5,4 \mathrm{ml}$ Blut, waren es beim traditionellen Verfahren $66,3 \mathrm{ml}$.

Fazit: In der Hochfrequenz-Chirurgie treten im Vergleich zu kalten Verfahren höhere Temperaturen auf, die Gewebeschäden und postoperative Schmerzen verursachen können. Ihr größter Pluspunkt ist die gleichzeitige Blutstillung. Die japanische Studie zeigt nun, dass sich im Bereich der Tonsillenchirurgie die Nachteile der elektrischen Instrumente durch anschließende Gewebekühlung vermeiden lassen, ohne den Vorteil des geringeren Blutverlustes einzubüßen. Dies könne, so die Autoren der Studie, besonders im pädiatrischen Bereich von Nutzen sein.

Dr. Robert Bublak

Horii A et al. Effects of cooling the pharyngeal muscosa after bipolar scissors tonsillectomy on postoperative pain. Acta Oto-Laryngol 2011; doi: 10.2109/00016489.2011.566580.

Postoperative Komplikationen gab es bei $8,3 \%$ der komplett thyreoidektomierten und bei $3,8 \%$ der hemithyreoidektomierten Patienten. Nur 2,6\% der Patienten wurden erneut eingewiesen. Es traten keine Todesfälle auf.

Fazit: Diese Zahlen sprechen dafür, dass die ambulante Thyreoidektomie, von einem erfahrenen Operateur durchgeführt, praktikabel und sicher zu sein scheint. Männer und Basedow-Patienten benötigen häufiger eine postoperative stationäre Betreuung.

Dr. Christine Starostzik

Hessmann C et al. Outpatient thyroidectomy: is it a safe and reasonable option? Am J Surg. 2011;201:565-8. 